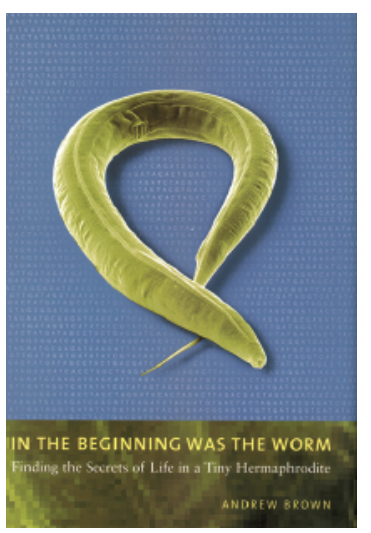

\title{
In the beginning was the worm
}

Finding the secrets of life in a tiny hermaphrodite

\author{
Andrew Brown \\ Columbia University Press, New York, New York, USA. 2003. \\ 244 pp. \$27.95. ISBN 0-231-13146-1 (hardcover).
}

Reviewed by Catherine A. Wolkow and Mark P. Mattson

E-mail:wolkowca@grc.nia.nih.gov

I n September of 1998, the press announced with great fanfare the completion of the first sequence of a multicellular organism. It was not the human genome, which would be completed two years later, or the mouse, rat, or fruit fly genomes. Rather, the 1998 announcement concerned a more modest organism, Caenorbabditis elegans, a small soildwelling nematode found in temperate climates worldwide that feeds on bacteria and slime mold. How C. elegans came to be the first multicellular organism to be sequenced in the genome race - a research project that garnered a Nobel Prize for illuminating genetic regulation and programmed cell death in the process - is the engaging tale related by Andrew Brown.

The book opens in the early 1960s, with Sydney Brenner and others at the Laboratory of Molecular Biology (LMB) in Cambridge, United Kingdom, debating their visions for the future path of molecular biology. Brenner believed that a molecular approach could be applied to studies of development and nervous system function and boldly proposed the genetic analysis of a simple eukaryote to accomplish these goals. The organism he ultimately chose for these studies, C. elegans, was selected for its rapid development, robust reproduction, anatomic transparency, and small size, conducive for detailed microscopic analysis.

Brown's description of the worm project reveals that Brenner had endeavored to construct a complete map of the worm on at least three different levels. The first level was the genetic map. This required the organism's genetic deconstruction. Brenner himself, along with the talented scientists he recruited, used chemical mutagenesis to isolate numerous mutations affecting development and movement. Following genetic techniques used in his earlier phage studies, Brenner could analyze the phenotypes of animals carrying combinations of these mutations to construct a genetic map for C. elegans. This genetic map shows the relative positions of the genes that led to the mutant phenotypes.

The second level of the map was an unprecedented four-dimensional description of embryonic and nervous system development. John White and Eileen Southgate, at LMB, described the anatomy and connectivity of the worm's 302-cell nervous system. Brown vividly presents the painstaking detail with which White and Southgate assembled electron micrographs from serial sections of the worm to determine the animal's nervous system connectivity. This map has allowed researchers to study specific neural pathways in detail. For example, scientists can identify the neural pathway by which signals from sensory neurons lead to a behavioral output, such as movement. Brown goes on to relate the work of John Sulston, at LMB, who described the complete developmental lineage of C. elegans: it is invariant, so that a specific cell's developmental fate is predictable. By comparing the lineage with the nervous system anatomy, Sulston and White could determine the developmental fates of every neuron precursor. The lineage analysis also revealed that certain cells were predictably lost during development. Studies of these lost cells by Bob Horvitz at LMB and, later, MIT led to the discovery of apoptosis in C. elegans and other species.

The third level of the map was the genome sequence, realized in 1998. The final chapters of Brown's book describe the scientific and political challenges faced by $C$. elegans researchers who worked on this task. Sulston and Alan Coulson at the LMB and Bob Waterston at Washington University in St. Louis coordinated this process. First, Sulston and Coulson assembled a physical map of the genome from cosmid and YAC libraries. They then coordinated the physical and genetic maps, allowing scientists to identify the precise physical position of cloned genes. With the physical map in hand, along with the cosmid and YAC libraries used in its construction, C. elegans was well positioned to be a trial genome project in preparation for sequencing the full human genome.

The biomedical sciences have made major advances in understanding human disease from studies of C. elegans. For example, Bcl-2 family members and caspases were first discovered in the worm. These proteins control the process of apoptosis, a form of cell death that is abnormally activated in many degenerative diseases and that is abnormally suppressed in many types of cancer. Drugs that target mammalian homologs of the worm proteins to inhibit or induce apoptosis are currently being developed for use in various human diseases. C. elegans is also proving to be a powerful tool to discover molecular mechanisms that regulate fundamental processes such as post-transcriptional gene silencing - a process called RNA interference that is now being exploited to develop novel gene-specific therapies for diseases.

Brown relates this engaging story in accessible language for scientists and laypeople alike. In an era when scientific storytelling has become commonplace, this book stands out for its lesson on independent thought. Scientists preferring more sophisticated organisms often challenged the worm project for its humbleness. Yet C. elegans has become one of the best-understood and most powerful systems in biology, due to the diligence and originality of the scientists described. Brown clearly relates the perseverance and vision of the first generation of worm scientists that led to these accomplishments. 